\title{
The numerical distance effect is task dependent
}

\author{
Liat Goldfarb • Avishai Henik • Orly Rubinsten • \\ Yafit Bloch-David • Limor Gertner
}

Published online: 23 June 2011

(C) Psychonomic Society, Inc. 2011

\begin{abstract}
Number comparison tasks produce a distance effect e.g., Moyer \& Landauer (Nature 215: 1519-1520, 1967). It has been suggested that this effect supports the existence of semantic mental representations of numbers. In a matching task, a distance effect also appears, which suggests that the effect has an automatic semantic component. Recently, Cohen (Psychonomic Bulletin \& Review 16: $332-336$, 2009) suggested that in both automatic and intentional tasks, the distance effect might reflect not a semantic number representation, but a physical similarity between digits. The present article (1) compares the distance effect in the automatic matching task with that in the intentional number comparison task and suggests that, in the latter, the distance effect does include an additional semantic component; and (2) indicates that the distance effect in the standard automatic matching task is questionable and that its appearance in previous matching tasks was based on the specific analysis and design that were applied.
\end{abstract}

Keywords Number processing, automaticity·Automatic processing, attention

\section{Goldfarb ( $\square)$}

The Center for the Study of Brain, Mind and Behavior (CSBMB), Princeton University,

Princeton, NJ 08540, USA

e-mail: lgoldfar@princeton.edu

\section{A. Henik · Y. Bloch-David • L. Gertner}

Department of Psychology and Zlotowski Center for

Neuroscience, Ben-Gurion University of the Negev,

Beer Sheva, Israel

\section{O. Rubinsten}

Edmond J. Safra Brain Research Center for the Study of Learning Disabilities, Department of Learning Disabilities,

University of Haifa,

Haifa, Israel
In 1967, Moyer and Landauer presented participants with two different digits between 1 and 9. Participants were asked to decide which digit, the right digit or the left, was numerically larger. It was found that response time (RT) increased as the distance between the digits decreased (e.g., RT was shorter for the pair "1 9" than for the pair "1 2"). This effect is known as the distance effect. The purpose of this article is to investigate whether the distance effect in this task is a result of the need to differentiate between overlapping semantic representations or whether it can only be attributed to the need to differentiate graphic similarity between digits (such as 8 and 9, both of which have circles on their tops). In addition, the aim of this article is to reinvestigate the automatic emergence of the distance effect in the matching task and, consequently, the theoretical notions that can be drawn from that task.

\section{The source of the distance effect: semantic or physical}

The distance effect can be explained by two components: physical and semantic. The semantic explanation takes into account the fact that digits are not just shapes but carry an important semantic content of quantity. The mental representation of a digit's semantic content is the source of the distance effect. Some theories suggest that numbers are semantically represented in analogical form. For example, the spatial mental number line theory suggests that a presented digit is converted to a mental representation along a spatial mental number line. When two digits are presented, they are converted into mental number representations on the same spatial mental line. This form of representation follows a very simple perception-like law: When two numbers on the same line are located close to each other (e.g., 1, 2), it is difficult to distinguish between 
them, since the encoding or the retrieval is impeded. As the number representations are located farther from each other, the distinction between them becomes easier. Because it is assumed that the efficiency of distinction is reflected in RT, Moyer and Landauer's (1967) distance effect can be explained by this theory (e.g., Dehaene \& Changeux, 1993; Dehaene, Dupoux \& Mehler 1990; Moyer, 1973). Other examples of the semantic representation of numbers that can explain the distance effect are the semantic network explanation (e.g., Whalen, 1996), the semantic coding theory (e.g., Banks, 1977), and memory-based theories that suggest that each number is attached to small or large attributes (e.g., Choplin \& Logan, 2005; Leth-Steensen \& Marley, 2000; Tzelgov, Meyer \& Henik 1992).

In contrast to the theories that explain the distance effect by semantic representation, it has been suggested that the effect can also be explained by physical similarity. Recently, Cohen (2009) suggested that numerical symbols might initially have been created in such a way that their physical shape reflects the quantities they represent. Correspondingly, the physical similarity must be correlated with the numerical distance. Hence, it is not clear whether the distance effect really reflects a semantic representation of numbers or only a physical similarity between the digits' shapes.

\section{The distance effect is found in both the comparison task and the matching task}

Whether the source of the distance effect is semantic or physical, it is very robust. Using Moyer and Landauer's (1967) standard intentional comparison task, a distance effect emerges for all single-digit numbers and for all the distances produced from these numbers. In fact, when participants have to decide which single digit is numerically larger, the distance effect always appears (e.g., Cohen Kadosh \& Henik, 2006; Gertner, Henik \& Cohen Kadosh 2009; Henik \& Tzelgov, 1982; Moyer \& Landauer, 1967), while in the standard comparison task, participants have to attend to the digit's meaning intentionally.

Dehaene and Akhavein (1995) suggested that the distance effect can also emerge automatically. Following Duncan and McFarland (1980), Dehaene and Akhavein (Experiment 1) asked participants to perform a single-digit number-matching task. They asked participants to decide whether two presented numbers were the same or different. The numbers appeared in the form of digits (e.g., 19 ) or words (e.g., one nine) or in a mixed form (e.g., one 9). One of the interesting findings in this study was that the distance effect emerged even when the participants performed the task relying only on the shape of the stimuli (as in the case in which the two digits were in the same notation). As Dehaene and Akhavein noted, "The distance effect remained highly significant even in the pure trials, in which the participants could conceivably have used physical or lexical identity as a cue for responding. . . . However, the participants evidently were not able to rely exclusively on presemantic levels of processing" (p. 319).

\section{The present study}

The present study had two purposes. The first aim of this study was to examine whether the distance effect in the standard intentional comparison task (e.g., Cohen Kadosh \& Henik, 2006; Gertner et al., 2009; Henik \& Tzelgov, 1982; Moyer \& Landauer, 1967) has a number semantic activation component. We have noted that Cohen (2009) suggested that the distance effect can be explained by physical similarity. Cohen's criticism regarding the role of the physical component in the emergence of the distance effect is applicable both to automatic tasks and to intentional tasks such as the standard intentional comparison tasks (e.g., Cohen Kadosh \& Henik, 2006; Gertner et al., 2009; Henik \& Tzelgov, 1982; Moyer \& Landauer, 1967). However, Cohen examined the physical component only with an automatic task. In his study, he used a variation of the standard automatic matching task in which he asked participants to indicate whether a certain digit between 1 and 9 was or was not the digit 5 . He found that in this task, the distance effect resulted from physical similarity between the digits. Can physical similarity entirely explain the emergence of the distance effect in an intentional task? This is the first topic that will be discussed in this article. We will suggest that in a nonautomatic task such as the standard comparison task, the distance effect does include a semantic component and that it cannot be attributed solely to digit similarity.

The second issue that will be discussed in this article is the distance effect that appears in the standard automatic matching tasks. We already noted that the distance effect found in the standard matching task in a design that was established by Dehaene and Akhavein (1995) is often taken as an indication for an automatic semantic representation of numbers (e.g., Eger, Sterzer, Russ, Giraud \& Kleinschmidt 2003; Otten, Sudevan, Logan \& Coles 1996; Xuan, Zhang, He \& Chen 2007). This is a surprising notion since, when the task requires matching nonnumerical stimuli, as in the case of color-word Stroop stimuli, automatic semantic processing is not observed. In the Stroop task, participants are asked to name the color of a color word or a color patch. Naming the color of an incongruent color word (e.g., the word blue written in a red color) is slower than naming the color of a colored patch or any other neutral stimulus. This finding is known as the Stroop interference effect, and it reflects the fact that word reading is an automatic process when one has to perform a color-naming task (Stroop, 
1935; see MacLeod, 1991, for an extensive review). Interestingly, in a version of the matching task using Stroop stimuli, an automatic interference is not observed. In this version, one has to decide whether the color of an incongruent, neutral, or congruent color word matches the color of another stimulus (e.g., participants have to respond "match" when the word green printed in red has to be matched to a red bar). Unlike the regular Stroop task, in the matching task, the word congruency does not influence participants' performance (e.g., Luo, 1999; Treisman \& Fearnley, 1969). Hence, it seems that a nonnumerical matching task does not produce automatic effects, but on the basis of the standard numerical version of the matching task established by Dehaene and Akhavein, it has been concluded in the literature that numbers are automatically activated (e.g., Eger et al., 2003; Otten et al., 1996; Xuan et al., 2007). We would like to comment that these conclusions need to be reevaluated due to the analyzing procedure that was established in Dehaene and Akhavein's study. This article created some conventions concerning the way matching tasks with single-digit numbers should be performed, and several rules that were established in the design of Experiment 1 were then followed by others (e.g., Ganor-Stern \& Tzelgov, 2008; Verguts \& Van Opstal, 2005). The design and the analyses in these automatic matching task experiments are different from the ones used in the standard intentional comparison task. It is not clear whether the distance effect that was found for single-digit numbers in the matching task experiments really reflects the common pattern characteristic of the distance effect that is found in other intentional task designs, such as the comparison task. The distance effect found in the standard automatic matching task and, consequently, the theoretical notions of the automatic distance effect that are derived from it need to be reexamined. The first limitation in the standard matching task design is that in all single-digit matching task studies, the statistical analysis has been performed on grouped distances (e.g., Dehaene \& Akhavein, 1995; Duncan \& McFarland, 1980; GanorStern \& Tzelgov, 2008; Verguts \& Van Opstal, 2005). In all those studies, the authors grouped together several distances in order to create a small-distance group and a large-distance group. When these groups were compared, it was found that RT was longer for the small distance than for the large distance. This finding led to the conclusion that the distance effect emerges in the matching task. Note that this is not exactly the normal distance effect that has been reported for the standard intentional comparison task. If it were the same distance effect, we would expect to find a distance effect not only between two grouped distances, but also within distances that were grouped together. This notion will be illustrated by an example from Dehaene and Akhavein's study. In Experiment 1, distance 1 was labeled as a small distance and distances 2 and greater were labeled as large distances. The distance effect that was reported in the article was measured by the comparison between those two distances. However, if indeed, in the automatic matching task, single-digit numbers produce the same distance effect that is produced by the intentional comparison task, then, for example, comparing distance 2 with distance 8 should also reveal a distance effect (as in the standard comparison task). From the current data, we cannot know whether this is indeed the case in the single-digit matching task.

In addition, we would like to note that there is another issue that might limit the conclusions that can be drawn from Dehaene and Akhavein's (1995, Experiments 1 and 2) design. When the authors tested whether numbers from 1 to 9 yielded a distance effect in a matching task, they presented the participants with various pairs composed of these digits. However, their analysis included only the pairs composed of the "stereotype" edge digits (the very small or very large digits) - that is, 1, 2, 8, and 9. The distance effect that they found was based only on these numbers. The other pairs were referred to as fillers and were not analyzed. Since this experiment set the standard for other matching experiments, Ganor-Stern and Tzelgov (2008) repeated this described design exactly. Similarly, in their matching task experiment, Verguts and Van Opstal (2005) analyzed only the digits $1,2,8$, and 9 , while not presenting the other single digits. If digits between 1 and 9 produce a distance effect when the automatic matching task is being performed, as in the case of the nonautomatic comparison task, we would expect to find a distance effect for all digits between 1 and 9. However, our review of the literature suggests that we cannot be sure that this is the case in the matching task.

\section{Experiment 1}

Experiment 1 had two main goals. The first aim was to examine the distance effect in both a standard intentional comparison task and a standard automatic matching task, with identical digits and analyses in the two tasks. Consequently, any difference between the results in the two tasks would reflect a difference between automatic and intentional semantic processing. Most important, if a difference was found, it would reflect a semantic component (that could not be attributed to the digits' physical shapes, as was suggested by Cohen, 2009). In other words, an interaction between tasks and distance would reflect a clean measure of a semantic distance effect with the perceptual shape similarity component held constant (since the physical component in the two tasks was the same). Second, if digits between 1 and 9 produce the distance 
effect, we would expect to find a distance effect for all pairs of digits that are commonly used in the comparison task. However, if previous matching task findings (e.g., Dehaene \& Akhavein, 1995; Ganor-Stern \& Tzelgov, 2008; Verguts \& Van Opstal, 2005) result from the specific design used, we might not find any evidence of a distance effect in the matching task.

Hence, Experiment 1 was composed of two parts. In Experiment 1a, we performed a replication of the regular comparison task, using balanced single-digit pairs that previously had produced a normal distance effect in this task (e.g., Cohen Kadosh \& Henik, 2006). After replicating previous studies and obtaining a normal distance effect in a comparison task, we performed Experiment $1 \mathrm{~b}$. In Experiment $1 \mathrm{~b}$, we used the same pairs of single-digit numbers that were used in Experiment 1a. However, instead of performing a comparison task, the participants were asked to perform a matching task. This allowed us to examine the interaction between those tasks and the distance effect, as well as the existence of the distance effect in a matching task.

\section{Experiment 1a}

\section{Method}

Participants Sixteen undergraduate students (13 females), native speakers of Hebrew, with normal or corrected-tonormal vision, participated in the experiment in partial fulfillment of course requirements.

Stimuli Each trial consisted of two digits, printed in bold in Courier New font, size 14. One digit appeared on the left of a computer screen, and one on the right, with 24 spaces separating the two digits. Following the study of Cohen Kadosh and Henik (2006), the digits 1-9, excluding 5, were coupled to create three numerical distances (1, 2, and 5), with the restraint that each digit appeared equally often for each distance. For distance 1, we used the pairs 1-2, 3-4, 6-7, and 8-9; for distance 2 , the pairs $1-3,2-4,6-8$, and 7-9; and for distance 5, the pairs $1-6,2-7,3-8$, and 4-9. In each block, each pair was presented two times with the smaller number on the left side and two times with the smaller number on the right side. Each participant was run with two experimental blocks of trials, each containing 48 trials (12 different pairs $\times 2$ sides $\times 2$ presentations $)$.

Procedure Data collection and stimuli presentation were controlled by a Compaq computer with an Intel Pentium III central processor. Stimuli were presented on a Compaq S510 monitor. Participants sat approximately $60 \mathrm{~cm}$ from the computer screen. A keyboard was placed on a table between the participants and the monitor. The participants were asked to press the "P" key on the keyboard if the right stimulus was numerically larger and to press the "Q" key if the left stimulus was numerically larger. Participants were instructed to respond as quickly as possible without making mistakes. Before the beginning of the experimental blocks, participants practiced on 24 experiment trials. These trials were identical to the ones in the experimental block.

All trials started with a fixation point (a black plus sign at the center of a white screen) for $300 \mathrm{~ms}$. Five hundred milliseconds after the fixation point disappeared, a pair of digits appeared at the center of the screen and remained visible until the participant responded or for 3,000 ms. The next trial began $1,000 \mathrm{~ms}$ after the disappearance of the stimulus. RT, in milliseconds, was measured by the computer from the stimulus onset until the participant's response.

\section{Results}

Mean RTs of correct responses were calculated for each participant in each condition. Participants that had more than $15 \%$ errors in one of the conditions were omitted from the analysis. One participant with 19\% errors fell into this category, and his data were omitted. A one-way analysis of variance (ANOVA) was applied to the rest of the data, with distance $(1,2$, and 5) as a within-participants factor. Mean RTs of correct responses and error rates (ERs) in the various conditions are presented in Table 1.

$R T$ analyses A significant main effect was found for distance, $F(2,28)=29.04, M S E=402, p<.001$. RT was longer for distance 1 than for distance $2, F(1,14)=31.70$, $M S E=257, p<.001$, and RT was longer for distance 2 than for distance $5, F(1,14)=9.12, M S E=414, p<.01$.

Error analyses A significant effect was found for distance, $F(2,28)=5.48, M S E=0.0005, p<.01$. The ER for distance 1 was nonsignificantly larger than that for distance $2, F<1$, and the ER for distance 2 was significantly larger than that for distance $5, F(1,14)=12.25, M S E=0.0005, p<.01$.

Table 1 Mean response times for correct responses in the various conditions of Experiment 1

\begin{tabular}{llll}
\hline Task & \multicolumn{2}{l}{ Distance } & \\
\cline { 2 - 4 } & 1 & 2 & 5 \\
\hline Regular comparison & $550(6.50 \%)$ & $517(5.61 \%)$ & $495(1.97 \%)$ \\
Matching & $568(2.34 \%)$ & $572(1.71 \%)$ & $573(1.25 \%)$ \\
\hline
\end{tabular}

Note. Response time is measured in milliseconds. Error rates are given in parentheses. 
Experiment $1 \mathrm{~b}$

\section{Method}

Twenty undergraduate students (16 females), native speakers of Hebrew, with normal or corrected-tonormal vision, participated in the experiment in partial fulfillment of course requirements. Each trial consisted of two digits, with one digit appearing on the left of a computer screen and one on the right. As in Experiment $1 \mathrm{a}$, for the nonmatched trials, the digits $1-9$, excluding 5 , were coupled to create three numerical distances: 1 (the pairs $1-2,3-4,6-7,8-9$ ), 2 (the pairs $1-3,2-4,6-8,7-$ 9 ), and 5 (the pairs 1-6, 2-7, 3-8, 4-9). Each digit appeared equally often on the left and on the right. In each block, each pair was presented 2 times with the smaller number on the left side and 2 times with the smaller number on the right side. Each participant was run with two experimental blocks of trials, each containing 48 trials $(12$ different pairs $\times 2$ sides $\times 2$ presentations). For the matched trials, the digits $1-9$, excluding 5 , were used to create eight pairs of identical digits. In each block, each pair was presented 6 times. Since there were two blocks in the experiment, the total number of experimental matched trials was 96 (8 digits pairs $\times 6$ presentations $\times 2$ blocks).

A keyboard was placed on a table between the participants and the monitor. The participants were asked to press the "P" key on the keyboard if the digits were matched and to press the "Q" key if the digits were not matched. Participants were instructed to respond as quickly as possible without making mistakes. Before the beginning of the experimental blocks, participants received 16 practice trials. These trials were selected randomly from the experimental block. All trials started with a fixation point (a black plus sign at the center of a white screen) for $300 \mathrm{~ms}$. Five hundred milliseconods after the fixation point disappeared, a pair of digits appeared at the center of the screen and remained visible until the participant responded or for 3,000 ms. RT, in milliseconds, was measured by the computer from the stimulus onset until the participant's response. The next trial began $500 \mathrm{~ms}$ after the disappearance of the stimulus. In all other aspects, Experiment 1a was the same as Experiment $1 \mathrm{~b}$.

\section{Results}

Mean RTs of correct responses were calculated for each participant in each condition. Since we were interested in the distance effect, we did not analyze the matched trials (distance 0). A one-way ANOVA was applied to the RT data, with distance $(1,2$, and 5) as a within-participants factor. Mean RTs of correct responses in the various conditions are presented in Table 1. Note that the pattern of RT is in the opposite direction to the pattern that is expected by the mental number line theory (RT for distance 1 is $4 \mathrm{~ms}$ shorter than that for distance 2, and RT for distance 2 is $1 \mathrm{~ms}$ shorter than that for distance 5). For the RT data, a significant main effect for distance was not found, $F<1$. A significant main effect for distance was also not found in the error analyses, $F(2,38)=1.00$, $M S E=0.0006, p=.377$.

In addition, we conducted a combined analysis of Experiments 1a and 1b. A two-way ANOVA was applied to the data, with distance $(1,2$, and 5) as a withinparticipants factor and experiment $(1 \mathrm{a}, 1 \mathrm{~b})$ as a betweenparticipants factor. A significant interaction effect was found, $F(2,66)=16.43, M S E=492, p<.001$. The differences in RT between distance 1 and distance 2 and between distance 2 and distance 5 were not the same between the two experiments, $F(1,33)=11.54, M S E=533$, $p<.01$, and $F(1,33)=4.97, M S E=450, p<.05$, respectively. Furthermore, we conducted another combined analysis of Experiments 1a and 1b, but this time with an equal number of participants in each experiment group. Hence, this time, only the first 15 participants in Experiment $1 \mathrm{~b}$ were analyzed. The two-way ANOVA that was applied to the data revealed the same pattern of results as in the previous analysis. A significant interaction effect was found between distance and experiment, $F(2,56)=11.47, M S E=$ $401, p<.001$. The differences in RT between distance 1 and distance 2 and between distance 2 and distance 5 were not the same between the two experiments, $F(1,28)=6.90$, $M S E=313, p<.025$, and $F(1,28)=5.34, M S E=452, p<$ .05 , respectively.

\section{Discussion}

The present experiment revealed that the distance effect that emerged in the comparison task (Experiment 1a) was different from the one that emerged in the matching task (Experiment 1b). The existence of an interaction between task and distance effect suggests that the distance effect has a significant component that relates purely to semantic activation (note that this effect is not influenced by the similarity in physical shape).

In addition, in this study, the standard distance effect emerged in the intentional comparison task but did not emerge in the standard automatic matching task. This suggests that the results from previous matching task studies that showed an automatic distance effect cannot be generalized, even within the same task, and that such a distance effect is restricted to the specific unusual design used. In Experiment 2, we continued to investigate the lack of the effect in the matching task. 


\section{Experiment 2}

Experiment 1 in Dehaene and Akhavein's (1995) study had a critical condition in which participants had to match between two digits. Experiment 2 herein replicated that condition. ${ }^{1}$ We presented participants with the same pairs of digits as those used by Dehaene and Akhavein in their study. We also performed exactly the same analysis as the authors performed; however, in addition, we performed two other analyses on the data. As we noted, Dehaene and Akhavein, Verguts and Van Opstal (2005), and GanorStern and Tzelgov (2008) reported that for pairs created from the numbers $1,2,8$, and 9 , response to distance 1 differed from responses to all other distances (since they grouped together the distances that were larger than 1 and then compared them with 1). Hence, the first analysis that we performed was identical to the one just described. In the second analysis, we were interested in examining whether the distance effect also would emerge when distances larger than 1 were not grouped together. Hence, we performed an ungrouped analysis on the same data. Finally, in the third analysis, we were interested in examining whether the distance effect was restricted only to the stereotype edge digits-1, 2, 8, and 9-or whether it was a general effect that emerged for all single-digit

\footnotetext{
${ }^{1}$ We would like to note that we replicated only the parts of interest from Dehaene and Akhavein's (1995, Experiment 1) method. As has already been mentioned, like the majority of distance effect studies, we were interested in studying only the mental representation of digits; hence, like all these other studies, we presented only pairs of digits. In contrast, Dehaene and Akhavein examined the distance effect under three conditions: a digit condition (as in our study), a number word condition (in which two number words were presented), and a mixed condition (in which one digit and one number word were presented). We had no reason to believe that presenting only the digit condition without presenting the other conditions in the same experiment would impair the replication of the digit condition itself. This is because, as in our study, Ganor-Stern and Tzelgov's (2008) matching task study replicated Dehaene and Akhavein's findings even when only pairs of digits were presented. A second difference between our procedure and Dehaene and Akhavein's procedure was the digits' presentation duration. While in our study the digit remained on the screen for 3,000 ms or until a participant response, in Dehaene and Akhavein's study the pairs appeared only for $200 \mathrm{~ms}$. Again, we performed this change because a rapid presentation of two digits is not the common procedure in the distance effect literature. We had no reason to believe that Dehaene and Akhavein's distance effect emerges only under short presentations and not under long ones, because the matching task studies that replicated Dehaene and Akhavein's study (Ganor-Stern \& Tzelgov, 2008; Verguts \& Van Opstal, 2005) presented the digits for a long duration. In fact, Ganor-Stern and Tzelgov addressed the issue of presentation duration in their study and found that the time the digits were presented did not influence the distance effect in the matching tasks.
}

numbers (as in the case of the standard intentional comparison task). Hence, we also analyzed the digits that were treated as fillers in previous studies.

\section{Method}

Fourteen undergraduate students (11 females), native speakers of Hebrew, with normal or corrected-to-normal vision, participated in the experiment in partial fulfillment of course requirements. As in Dehaene and Akhavein's (1995) study, the digits $1,2,8$, and 9 were coupled to create five numerical distances: 0 (the pairs 1$1,2-2,8-8,9-9$ ), 1 (the pairs $1-2$ and 8-9), 6 (the pair 28), 7 (the pair 2-9), and 8 (the pair 1-9). Following Dehaene and Akhavein's design, for the digits 1, 2, 8, and 9 , the pairs with the distances 0 and 1 appeared twice as often as the pairs with distances 6,7 , and 8 . In each block, the pairs with distance 0 and 1 appeared 8 times each, and the pairs with the distances 6-8 appeared 4 times each. In addition, Dehaene and Akhavein used 40 filler trials in each block. These fillers were created from the digits 3-7. In our experiment, we also employed all possible two-digit pairs created from the digits 3-7. The following distances were created: 0 (the pairs $3-3,4-4$, $5-5,6-6,7-7$ ), 1 (the pairs $3-4,4-5,5-6,6-7$ ), 2 (the pairs 3-5, 4-6, 5-7), 3 (the pairs 3-6, 4-7), and 4 (the pair 3-7). Each digit appeared equally often on the left and on the right. To increase the proportion of the matched responses, all pairs with the distance 0 were presented 2 additional times. Overall, we created 40 filler trials $(15$ pairs $\times 2$ sides +10$)$ with the numbers $3-7$. Unlike in Dehaene and Akhavein's study, we were interested in analyzing these pairs, so we presented each one of the 40 combinations 4 times in each block. There were two blocks in the experiment and 16 practice trials; overall, the total number of the trials in the experiment was 512.

\section{Results and discussion}

Mean RTs of correct responses were calculated for each participant in each condition. Mean RTs of correct responses for the pairs created from the numbers $1,2,8$, and 9 for the various distances and ERs are presented in Table 2 and for the numbers 3, 4, 5, 6, and 7 in Table 3 . Recall that Dehaene and Akhavein (1995), Verguts and Van Opstal (2005), and Ganor-Stern and Tzelgov (2008) reported that for pairs created from the numbers $1,2,8$, and 9, distance 1 differed from distances larger than 1 (since they grouped together distances larger than 1 and then compared them with 1). We performed the same analysis 
Table 2 Mean response times for correct responses for the pairs created by the numbers $1,2,8$, and 9 for the various distances in Experiment 2

Distance

\begin{tabular}{llll}
\hline 1 & 6 & 7 & 8 \\
\hline $588(2.90 \%)$ & $578(0 \%)$ & $558(1.22 \%)$ & $541(0.44 \%)$ \\
\hline
\end{tabular}

Note. Response time is measured in milliseconds. Error rates are given in parentheses.

and found the same significant effect, $F(1,13)=21.65$, $M S E=409, p<.01 .^{2}$

We then examined the effect in all the other single-digit numbers (the digits that were considered as fillers). From Table 3, it is clear that for the pairs created from the numbers $3,4,5,6$, and 7, the mean RT pattern does not fit the expected distance effect. For distances 1, 2, and 3, mean RT was longer as the distance increased. Mean RT was shortest for distance 4 .

The ANOVA revealed a significant main effect, $F(3,39)=$ 9.3205, $M S E=738, p<.001$. However, as we noted, this cannot be due to the normal distance effect. For distances 1, 2 , and 3, RT nonsignificantly lengthened as the distance increased (which is the opposite pattern of the normal distance effect, but note that it was insignificant): $F(1,13)=$ $1.46, M S E=510, p=.247$ for the difference between distances 1 and 2, and $F<1$ for the difference between distances 2 and 3. RT was shorter to distance 4 than to all other distances, $F(1,13)=28.91, M S E=636, p<.001$. An error analysis performed on these pairs did not reveal a significant main effect in the error rate, $F<1$.

Similar to Verguts and Van Opstal (2005), and GanorStern and Tzelgov (2008), we also replicated Dehaene and Akhavein's (1995) results, since we found that, for pairs created from the numbers $1,2,8$, and 9, RT to distance 1 differed from all other distances when the latter were grouped together. This supposedly indicates that we did not have any specific problems in our experiment, since we

\footnotetext{
${ }^{2}$ We also examined the distance effect with the digits $1,2,8$, and 9, but, this time, ungrouped, such that the distances could be larger than 1 (i.e., we compared all the distances that could be created from these digits-distances 1, 6, 7, and 8). For the pairs created from the numbers $1,2,8$, and 9 , we found a significant main effect for distance, $F(3,39)=8.28, M S E=741, p<.001$. The pattern of mean RT fit with the normal distance effect (i.e., RT to distance 1 was longer than that to distance 6 , RT to distance 6 was longer than that to distance 7 , and RT to distance 7 was longer than that to distance 8). However, only the difference between distances 6 and 7 was significant, $F(1,13)=5.8$, $M S E=490, p<.05$. The differences between distances 1 and 6 and between 7 and 8 were not significant, $F(1,13)=1.86, M S E=380, p=$ .195 , and $F(1,13)=1.36, M S E=1,434, p=.26$, respectively.
}

Table 3 Mean response times for correct responses for the pairs created by the numbers $3,4,5,6$, and 7 for the various distances in Experiment 2

Distance

\begin{tabular}{llll}
\hline 1 & 2 & 3 & 4 \\
\hline $574(1.45 \%)$ & $584(1.93 \%)$ & $591(2.00 \%)$ & $541(1.33 \%)$ \\
\hline
\end{tabular}

Note. Response time is measured in milliseconds. Error rates are given in parentheses.

were able to replicate previous findings of others perfectly. However, when we analyzed the other numbers-3, 4, 5, 6, and 7-(the numbers that were not analyzed in Dehaene \& Akhavein, 1995, and Ganor-Stern \& Tzelgov, 2008, and did not appear in Verguts \& Van Opstal, 2005), we found no significant effect for distances, and even the insignificant pattern of the mean RT could not fit with the spatial mental number line theory (RT was nonsignificantly shorter for distance 1 than for distance 2 and RT was nonsignificantly shorter for distance 2 than for distance 3 ).

The present pattern of results makes it hard to argue for a general claim that single-digit numbers automatically generate the distance effect when a matching task is performed.

\section{General discussion}

Let us first summarize our main results. In Experiment 1a, we found a regular distance effect in a standard intentional comparison task, which replicated previous comparison task findings (e.g., Cohen Kadosh \& Henik, 2006; Gertner et al., 2009; Henik \& Tzelgov, 1982; Moyer \& Landauer, 1967). However, in Experiment 1b, when the comparison task was changed to a matching task, the same distance effect did not appear. In fact, the distance effect disappeared for the matching task. A significant interaction was found between those tasks and distance. Experiment 2 employed another matching task in which we presented the same pairs of digits as in Dehaene and Akhavein's (1995) single-digit experiment (Experiment 1) and analyzed it as they had analyzed it. That is, we analyzed only the edge digits $(1,2,8$, and 9) and then compared distance 1 with all other distances grouped together. Using this method, we replicated Dehaene and Akhavein's study and all other studies that used this specific method (e.g., Ganor-Stern \& Tzelgov, 2008; Verguts \& Van Opstal, 2005). We also found that for those pairs, response was slower to distance 1 than to all other distances when the latter were grouped together. When we performed another 
analysis in which we analyzed the numbers $3,4,5,6$, and 7 (the numbers that were not analyzed in Dehaene \& Akhavein's [1995] and Ganor-Stern \& Tzelgov's [2008] study and did not appear in Verguts \& Van Opstal's [2005] study), we did not find a pattern similar to the normal distance effect.

As we noted in the introduction, this article deals with two issues. The first one concerns the interpretation of the distance effect found in intentional tasks, such as the widely used comparison task. Cohen (2009) suggested that digits' physical shapes are correlated with the quantities they represent and, consequently, the digits' physical shapes are correlated with the distance effects observed in the literature. Hence, it is not clear whether the distance effect in the standard intentional comparison task really reflects semantic representation of numbers on a spatial mental line or only physical similarity between digits. The interaction found in Experiment 1 between the matching task and the comparison task suggests that the distance effect found in the intentional comparison task has a semantic representation component and that this distance effect cannot reflect merely physical similarity between digits (i.e., since the physical component in the two task was the same, the significant, larger distance effect in the comparison task must reflect a clean measure of a semantic distance effect).

Second, we suggest that the standard matching task used by several researchers to demonstrate an automatic distance effect might not provide strong evidence for the existence of a standard distance effect. This was indicated when we showed that previous conclusions drawn from the standard automatic matching tasks are not general and are limited to the unusual analysis performed on the standard matching tasks.

Number semantic representation, automaticity, and task demand

Dehaene and Akhavein's (1995) task tends to be perceived in the literature as evidence for a task that supposedly demonstrates automatic activation of the semantic component of numbers (e.g., Eger et al., 2003; Otten et al., 1996; Xuan et al., 2007). Automatic processes have several definitions. An automatic process is commonly defined as one that is autonomic, ballistic, and effortless and that one is unaware of (e.g., Hasher \& Zacks, 1979; Logan, 1992; Posner, 1978). Tzelgov (1997, 1999) suggested an empirical test to diagnose an automatic process. He proposed that if a process is not part of the task requirements and yet interferes with another process, the former process is an automatic process. As was already noted by Dehaene and Akhavein, in order to match between two different digits, one does not need to process any semantic aspect of the digits. However, if the matching task does trigger the semantic components of numbers, it means that this is an automatic representation. As we noted, Cohen (2009) recently challenged this view and showed that in another task, in which one did not have to attend to the digits' semantic level, automatic activation was not found. He suggested that the distance effect can actually be attributed to the digit's physical components. This suggestion can also be applied to nonautomatic tasks. However, our study suggests that when we compare the standard matching task, which is an automatic task, with the standard comparison task, which is an intentional task, an indication for the semantic component in the intentional task is observed.

In addition, the normal distance effect, which, as we noted, is assumed to represent automatic semantic activation by some (e.g., Dehaene \& Akhavein, 1995; Eger et al., 2003; Ganor-Stern \& Tzelgov, 2008; Otten et al., 1996; Verguts \& Van Opstal, 2005; Xuan et al., 2007), does not appear when we analyze the standard automatic matching task correctly. The different results that were obtained for the intentional and automatic tasks suggest that number semantic activation is not absolutely automatic but depends, to some extent, on task demands. This notion places automatic processing of numbers in a much wider context. It is well documented that some other processes are automatic only to some extent and are mainly task dependent. A classic example for this is the word-reading process in a Stroop task. In the introduction, we noted that in the Stroop task in which participants are asked to name the color of a color word or a color patch, the word is processed automatically (see MacLeod, 1991, for an extensive review). It is well documented that this interference is task dependent (e.g., Durgin, 2000; Pritchatt, 1968; Sugg \& McDonald, 1994; Virzi \& Egeth, 1985) and in a version of the matching task using Stroop stimuli, an automatic interference is not observed. Unlike the regular Stroop task, but similar to the one in the present study, in a matching task the word does not influence participants' performance (e.g., Luo, 1999; Treisman \& Fearnley, 1969). What role does the task demand play in the emergence of automatic activation, and why do certain tasks produce automatic activation and others do not? A Stroop color-naming task produces automatic word reading, and a number comparison task produces automatic semantic distance effect representation. Nevertheless, the automatic matching task does not produce either of these, whether the automatic semantic processing of numbers or the automatic processing of words is examined. What role does task demand play in the disappearance or the appearance of the effect? One possible framework that can deal with these findings revolves around theories that attribute to the system abilities that are responsible for the adjustment of input that enters the system according to task demand (e.g., Cohen, Dunbar \& McClelland, 1990; Roelofs, 2003). This notion was originally presented by Treisman in 1969 in the analyzer theory. According to 
this theory, each attribute, such as color, size, or shape, is processed by a specific analyzer. According to the task demand, each analyzer is either opened or switched off (i.e., processes or does not process an attribute). When an analyzer is needed in order to fulfill a task, one cannot switch off that analyzer. Hence, input from the opened analyzer is active and can intrude on the performance of the task. Treisman noted that when the task is matching between two colors (as in the Stroop matching task), it does not entail word processing in order to execute the correct response. Hence, perceptual processing of the irrelevant word is reduced by closing the word analyzer, and the word does not automatically intrude on color matching. Similarly, we can generalize from the wordreading process in the Stroop task to the number-processing tasks. When the task is matching between two digits, as in our study, or making a physical shape judgment related to another mental representation, as in Cohen (2009) study, the task does not entail deep quantity processing in order to execute the correct response. Hence, perceptual processing of quantity is reduced by task demand, and no automatic semantic representation is observed. However, when the task requires the quantity analyzer to be opened, such as in the comparison task, a distance effect with a semantic component is observed.

To sum up, it seems that the semantic activation of numbers is not an absolutely automatic process. Unlike some suggestions in the literature (e.g., Dehaene \& Akhavein, 1995; Eger et al., 2003; Ganor-Stern \& Tzelgov, 2008; Otten et al., 1996; Verguts \& Van Opstal, 2005; Xuan et al., 2007), the judgment of the physical shape of digits is not enough to trigger the semantic distance effect. In order for the semantic component to be activated and for the semantic distance effect to emerge, the semantic component has to be triggered to some extent by the task demand.

Authors Note This work was conducted as part of the research for the Center for the Study of the Neurocognitive Basis of Numerical Cognition, supported by the Israel Science Foundation (Grant 1664/08) in the framework of their Centers of Excellence.

\section{References}

Banks, W. P. (1977). Encoding and processing of symbolic information in comparative judgments. In G. H. Bower (Ed.), The psychology of learning and motivation (Vol. 2, pp. 101-159). New York: Academic.

Choplin, J. M., \& Logan, G. D. (2005). A memory-based account of automatic numerosity processing. Memory and Cognition, 33, $17-28$.

Cohen, D. J. (2009). Integers do not automatically activate their quantity representation. Psychonomic Bulletin and Review, 16, $332-336$.
Cohen Kadosh, R., \& Henik, A. (2006). A common representation for semantic and physical properties: A cognitive-anatomical approach. Experimental Psychology, 53, 87-94.

Cohen, J. D., Dunbar, K., \& McClelland, J. L. (1990). On the control of automatic processes: A parallel distributed processing account of the Stroop effect. Psychological Review, 97, 332-361.

Dehaene, S., \& Akhavein, R. (1995). Attention, automaticity and levels of representation in number processing. Journal of Experimental Psychology. Learning, Memory, and Cognition, 21, 314-326.

Dehaene, S., \& Changeux, J. P. (1993). Development of elementary numerical abilities: A neuronal model. Journal of Cognitive Neuroscience, 5, 390-407.

Dehaene, S., Dupoux, E., \& Mehler, J. (1990). Journal of Experimental Psychology. Human Perception and Performance, 16, 626-641.

Duncan, E. M., \& McFarland, C. E. (1980). Isolating the effects of symbolic distance and semantic congruity in comparative judgments: An additive-factors analysis. Memory and Cognition, 8, $612-622$.

Durgin, F. H. (2000). The reverse Stroop effect. Psychonomic Bulletin and Review, 7, 121-125.

Eger, E., Sterzer, P., Russ, M. O., Giraud, A. L., \& Kleinschmidt, A. (2003). A supramodal number representation in human intraparietal cortex. Neuron, 37, 719-725.

Ganor-Stern, D., \& Tzelgov, J. (2008). Across-notation automatic numerical processing. Journal of Experimental Psychology. Learning, Memory, and Cognition, 34, 430-437.

Gertner, L., Henik, A., \& Cohen Kadosh, R. (2009). When 9 is not on the right: Implications from number--form synesthesia. Consciousness and Cognition, 18, 366-374.

Hasher, L., \& Zacks, R. (1979). Automatic and effortful processes in memory. Journal of Experimental Psychology. General, 108, $356-388$.

Henik, A., \& Tzelgov, J. (1982). Is three greater than five: The relation between physical and semantic size in comparison tasks. Memory and Cognition, 10, 389-395.

Leth-Steensen, C., \& Marley, A. A. J. (2000). A model of response time effects in symbolic comparison. Psychological Review, 107, $62-100$.

Logan, G. D. (1992). Attention and preattention in theories of automaticity. The American Journal of Psychology, 105, 317-339.

Luo, C. R. (1999). Semantic competition as the basis of Stroop interference: Evidence from color-word matching tasks. Psychological Science, 10, 35-40.

MacLeod, C. M. (1991). Half a century of research on the Stroop effect: An integrative review. Psychological Bulletin, 109, 163-203.

Moyer, R. S. (1973). Comparing objects in memory: Evidence suggesting an internal psychophysics. Perception \& Psychophysics, 13, 180-184.

Moyer, R. S., \& Landauer, T. K. (1967). Time required for judgments of numerical inequality. Nature, $215,1519-1520$.

Otten, L. J., Sudevan, P., Logan, G. D., \& Coles, M. G. H. (1996). Magnitude vs. parity in numerical judgements: Event-related brain potentials implicate response conflict as the source of interference. Acta Psychologica, 94, 21-40.

Posner, M. I. (1978). Chronometric explorations of mind. Hillsdale: Erlbaum.

Pritchatt, D. (1968). An investigation into some of the underlying associative verbal processes of the Stroop colour effect. Quarterly Journal of Experimental Psychology, 20, 351-359.

Roelofs, A. (2003). Goal-referenced selection of verbal action: Modeling attentional control in the Stroop task. Psychological Review, 110, 88-125.

Stroop, J. R. (1935). Studies in interference in serial verbal reactions. Journal of Experimental Psychology, 18, 643-661. 
Sugg, M. J., \& McDonald, J. E. (1994). Time course of inhibition in color-response and word-response versions of the Stroop task. Journal of Experimental Psychology. Human Perception and Performance, 20, 647-675.

Treisman, A. M. (1969). Strategies and models of selective attention. Psychological Review, 76, 282-299.

Treisman, A. M., \& Fearnley, S. (1969). The Stroop test: Selective attention to colours and words. Nature, 222, 437-439.

Tzelgov, J. (1997). Specifying the relations between automaticity and consciousness: A theoretical note. Consciousness and Cognition, 6, 441-451.

Tzelgov, J. (1999). Automaticity and processing without awareness. Psyche, 5. Retrieved from http://psyche.cs.monash.edu.au/v5/ psyche-5-05-tzelgov.html.
Tzelgov, J., Meyer, J., \& Henik, A. (1992). Automatic and intentional processing of numerical information. Journal of Experimental Psychology. Learning, Memory, and Cognition, 18, 166-179.

Verguts, T., \& Van Opstal, F. (2005). Dissociation of the distance effect and size effect in one-digit numbers. Psychonomic Bulletin and Review, 12, 925-930.

Virzi, R. A., \& Egeth, H. E. (1985). Toward a translational model of Stroop interference. Memory and Cognition, 13, 304-319.

Whalen, J. (1996). The influence of semantic number representation on arithmetic fact retrieval (Unpublished doctoral dissertation). Baltimore: Johns Hopkins Universitiy.

Xuan, B., Zhang, D., He, S., \& Chen, X. (2007). Larger stimuli are judged to last longer. Journal of Vision, 7, 1-5. 